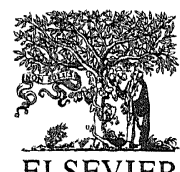

\title{
Small weight bases for Hamming codes ${ }^{1}$
}

\author{
John Tromp ${ }^{\mathrm{a}, *}$, Louxin Zhang ${ }^{\mathrm{a}}$, Ying Zhao ${ }^{\mathrm{b}}$ \\ a Department of Computer Science, University of Waterloo, Waterloo, Ont., Canada N2L $3 G 1$ \\ bepartment of Mathematics, Shanxi Teacher's University, Linfei, 041004 Shanxi, China
}

\begin{abstract}
We present constructions of bases for a Hamming code having small width and height, i.e. number of $1 \mathrm{~s}$ in each row and column in the corresponding matrix. Apart from being combinatorially interesting in their own right, these bases also lead to improved embeddings of a hypercube of cliques into a same-sized hypercube.
\end{abstract}

\section{Introduction}

Let $n=2^{k}-1, k \geqslant 2$, and let $A_{k}$ be the $k$ by $n$ matrix over $G F(2)$ whose $i$ th column, for $1 \leqslant i \leqslant n$, is the $k$-bit binary representation of $i$. For example,

$$
A_{3}=\left(\begin{array}{lllllll}
1 & 0 & 1 & 0 & 1 & 0 & 1 \\
0 & 1 & 1 & 0 & 0 & 1 & 1 \\
0 & 0 & 0 & 1 & 1 & 1 & 1
\end{array}\right) .
$$

We denote by $C_{k}$ the nullspace of $A_{k}$, i.e. the set of $n$-vectors $x$ with $A_{k} \cdot x=0^{k}$. We are interested in finding a basis of the nullspace, $C_{k}$, of $A_{k}$, that has small height and width. The height of a set of vectors is defined as the maximum number of ones in any vector, while width is defined as the maximum over all $n$ positions, of the number of vectors in the set having a 1 in that position. A basis of height $h$ and width $w$ is called a $(h, w)$-basis. The pair $(h, w)$ is called the weight.

Low weight bases for the nullspace $C_{k}$ have applications in coding theory [8], combinatorial designs [2], network embeddings [1,6], and distributing resources in hypercube computers [10]. In fact, $C_{k}$ is a one-error-correcting code which was first discovered by Hamming [5] for words of length $2^{k}-k-1$. More precisely, Hamming proved that the words of length $2^{k}-k-1$ can be encoded as words of length $2^{k}-1$ so that each word has Hamming distance at most 1 to exactly one codeword.

\footnotetext{
This work was supported in part by an NSERC International Fellowship and ITRC.

* Correspondence address: CWI, P.O. Box 94079, 1090 GB Amsterdam, The Netherlands. E-mail: tromp@cwi.nl.
} 
Recently, bases for $C_{k}$ were shown to be useful for hypercube embeddings. An embedding of a network $G$ into a network $H$ consists of an assignment of nodes of $G$ to nodes of $H$ and a mapping from edges of $G$ onto paths in $H$. Desirable properties of an embedding are small load (maximum number of nodes of $G$ assigned to the same node in $H$ ), low dilation (maximum length of a path that an edge is mapped to) and low congestion (maximum number of paths using an edge). In [1], Aiello and Leighton discovered that for any $k>0$, a $(h, w)$-basis for $C_{k}$ induces a one-toone embedding of a hypercube of cliques $H_{2^{k}-k} \otimes K_{k}$ in a same-sized hypercube $H_{2^{k}}$ with dilation $h$ and congestion $2 w+2$. Moreover, this embedding is useful in finding efficient embeddings of (dynamic) binary trees in the hypercube and reconfigurations of the hypercube around faults.

Although the existence of a height 3 basis for $C_{k}$ is well known, the existence question for a (3,3)-basis is open ([6, p. 430]). Towards this problem, only weak results were obtained in $[1,6,9,12]$. In this paper, we present two classes of bases with small weight, which improve the existing bounds on weight. In Section 2, we present a $(3,5)$-basis for $C_{k}$ that has a very simple structure.

There are many constructions of codes from the incidence matrices of graphs, designs, etc. (for example, see [3,9]). Using the approach observed in [9], we construct a class of $(3,4)$-bases in Section 3. As a consequence, we obtain a better one-to-one embedding of a hypercube of cliques into a same-sized hypercube, with dilation 3 and congestion 10 .

Finally, we propose a construction of (3,3)-bases. In [1], Aiello and Leighton observed that a primitive trinomial of degree $k$ induces a $(3,3)$-basis for $C_{k}$. But, primitive trinomials do not always exist. This observation is generalized in Section 4. We show that the existence of a trinomial $f(x)$ such that $\operatorname{gcd}\left(f(x), x^{2^{k}-1}+1\right)$ is primitive of degree $k$ implies a $(3,3)$-basis for $C_{k}$. We present results of computations supporting our conjecture that such trinomials always exist.

\section{A simple construction of a $(3,5)$-basis}

Note that the rank of $A_{k}$ equals $k$. It follows that $C_{k}$ has rank $n-k$, and that a basis for it consists of $n-k$ linearly independent vectors. We identify a boolean $n$-vector with its support, i.e. the set of positions (as non-zero boolean $k$-vectors) where it has a 1. For example, the support of $(0100101)$ is $\{010,101,111\}$. The product $A_{k} \cdot\{u, v, w\}$ is easily seen to equal the sum over $\mathrm{GF}(2)$ (bitwise exclusive-or) of $u, v$, and $w$. E.g. $A_{3} \cdot\{010,101,111\}=010 \oplus 101 \oplus 111=0^{3}$. To better visualize the exclusive-or operation, we sometimes write the vectors in the support below each other with the bits aligned:

$$
\left\{\begin{array}{lll}
0 & 1 & 0 \\
1 & 0 & 1 \\
1 & 1 & 1
\end{array}\right\} .
$$


For a bit $b$, we denote by $\bar{b}$ its complement $b \oplus 1$. For a binary string/vector $x,|x|$ denotes the length of $x$.

A basis of $C_{k}$ is constructed as follows. For $x \in\{0,1\}^{i}$, and $i+p+2 \leqslant k$, let $b_{x . p}$ be the vector

$$
\left\{\begin{array}{ccccccccc}
0^{k-i-p-2} & 1 & x_{1} & x_{2} & \ldots & x_{i-1} & x_{i} & 1 & 0^{p} \\
0^{k-i-p-2} & 0 & 1 & x_{1} & \ldots & x_{i-2} & \overline{x_{i}} & 1 & 0^{p} \\
0^{k-i-p-2} & 1 & \overline{x_{1}} & x_{1,2} & \ldots & x_{i-2, i-1} & 1 & 0 & 0^{p}
\end{array}\right\},
$$

where we write $x_{i, j}$ for $x_{i} \oplus x_{j}$. For definiteness, we have for the cases $i=0,1$ :

$$
b_{k, p}=\left\{\begin{array}{llll}
0^{k-p-2} & 1 & 1 & 0^{p} \\
0^{k-p-2} & 0 & 1 & 0^{p} \\
0^{k-p-2} & 1 & 0 & 0^{p}
\end{array}\right\}, \quad b_{x_{1}, p}=\left\{\begin{array}{ccccc}
0^{k-p-3} & 1 & x_{1} & 1 & 0^{p} \\
0^{k-p-3} & 0 & \overline{x_{1}} & 1 & 0^{p}, \\
0^{k-p-3} & 1 & 1 & 0 & 0^{p}
\end{array}\right\} .
$$

Note that $A_{k} \cdot b_{x, p}=0^{k}$, so that any $b_{x, p}$ is in $C_{k}$.

Our proposed basis simply consists of the set $B$ of all $b_{x, p}$. We must check that these vectors are indeed independent and that we have the right number of them.

To see the latter, partition $B$ into $k-1$ sets $B_{p}$, and each $B_{p}$ into $k-p-1$ sets $B_{p, i}$, containing all $b_{x, p}$ with $|x|=i$. Clearly, different pairs $(x, p)$ define different vectors. Thus, the size of $B$ is

$$
\sum_{p=0}^{k-2} \sum_{i=0}^{k-2-p} 2^{i}=\sum_{p=0}^{k-2}\left(2^{k-1-p}-1\right)=2^{k}-2-(k-1)=n-k
$$

Thus, to prove that $B$ is a basis, it remains to show that its elements are linearly independent.

\subsection{Independence}

Consider any nonempty subset $C$ of $B$. We prove independence by showing that the sum of all vectors in $C$ is not $0^{k}$.

Let $p$ be minimal such that $C \cap B_{p} \neq \emptyset$ and for this $p$, let $i$ be maximal such that $C \cap B_{p, i} \neq \emptyset$, say $b_{x, p} \in C \cap B_{p, i}$. By definition, $b_{x, p}$ has $0^{k-i-p-2} 1 \times 10^{p}$ in its support. For any other $b_{x^{\prime}, p^{\prime}}$ to have $0^{k-i-p-2} 1 \times 10^{p}$ in its support, would require either $p^{\prime}=p-1$ or $\left|x^{\prime}\right|=|x|+1$, so by minimality of $p$ and maximality of $i$, such a $b_{x^{\prime}, p^{\prime}}$ cannot be in $C$. Since $b_{x, p}$ is thus the only vector in $C$ with $0^{k-i-p-2} 1 \times 10^{p}$ in its support, the sum of all vectors in $C$ also has $0^{k-i-p-2} 1 \times 10^{p}$ in its support and hence is not $0^{k}$.

\subsection{Height and width}

The height of $B$ is obviously 3 , since each vector $b_{x, p}$ has exactly 3 one bits. We claim that the width of $B$ is at most 5 . To see this, consider any position $z$. If $z$ is of 
the form $0^{k-q-1} 10^{q}$ then it appears only in the support of $b_{k, q}, b_{k, q-1}$ (if $q>0$ ), and $b_{1, q}$. Hence, the width at such positions is no more than 3 .

Otherwise, $z$ is of the form $0^{k-j-q-2} 1 y_{1} y_{2} \ldots y_{j} 10^{4}$. Consider the $b_{x, p}$ that have this $z$ in their support. We necessarily have one of the following three cases.

1. $z=1 \times 10^{p}$. This implies $p=q$ and $x=y$, and so accounts for one $b_{x, p}$.

2. $z=1 x_{1} \ldots x_{i-2} \bar{x}_{i} 10^{p}$. This implies $p=q, x_{1: i-2}=y_{1: j-1}$ and $x_{i}=\overline{y_{j}}$, and so accounts for two $b_{x, p}\left(x_{i-1}\right.$ can be 0 or 1$)$.

3. $z=1 \overline{x_{1}} x_{1,2} \ldots x_{i-2, i-1} 100^{p}$. This implies $p=q-1$ and $x_{1}=\overline{y_{1}}, x_{2}=y_{2} \oplus x_{1}=$ $\overline{y_{1}} \oplus y_{2}, x_{3}=y_{3} \oplus x_{2}=\overline{y_{1}} \oplus y_{2} \oplus y_{3}, \ldots, x_{i-1}=\overline{y_{1}} \oplus y_{2} \oplus \cdots \oplus y_{j}$, and so accounts for two $b_{x, p}\left(x_{i}\right.$ can be 0 or 1$)$.

In total we find that at most five $b_{x, p}$ can have a one in position $z$, as claimed.

\section{A $(3,4)$ basis}

While the $(3,5)$ basis may be preferred in some applications for its simplicity, we can get a better $(3,4)$ basis by combining results from finite fields with an inductive construction based on finding Hamiltonian paths in complete bipartite graphs.

We start with the empty base $B_{1}$ for the null space $C_{1}=\{0\}$ of $A_{1}$, which is the 1 by 1 matrix (1). Next we explain how to extend $B_{k}$ to a basis $B_{k+1}$ for the null space $C_{k+1}$. A subset $B_{k}^{\prime}$ of $2^{k}-1-k$ vectors in $B_{k+1}$ will be derived from the $2^{k}-1-k$ vectors in $B_{k}$. Namely, for each vector $\{u, v, w\}$ in $B_{k}$, where $u, v, w \in\{0,1\}^{k}$, we put $\{0 u, 0 v, 0 w\}$ into $B_{k}^{\prime}$.

We form $B_{k+1}$ as the union of $B_{k}^{\prime}$ and a set $B$ of $2^{k}-1$ more vectors, to get the required number of $2^{k}-1-k+2^{k}-1=2^{k+1}-1-(k+1)$ vectors. These vectors will have a support consisting of one position in $X=01\{0,1\}^{k-1}$ and one in each $Y_{i}=1 i\{0,1\}^{k-1}, i=0,1$. Note that, for such a vector $\left\{01 x, 10 y_{0}, 11 y_{1}\right\}$ to be in the nullspace, it must satisfy $x=y_{0} \oplus y_{1}$, so that it is determined by just the pair $\left(10 y_{0}, 11 y_{1}\right) \in Y_{0} \times Y_{1}$. Our problem can thus be seen as the selection of $2^{k}-1$ edges in the complete bipartite graph $G$ on $Y_{0} \cup Y_{1}$. We will consider $X$ to be a set of colors and say that an edge between $10 y_{0}$ and $11 y_{1}$ has color $01\left(y_{0} \oplus y_{1}\right) \in X$. Getting a low width basis corresponds to minimizing the maximum degree of any vertex and simultaneously minimizing the maximum number of edges of any color. Our construction is based on finding a Hamiltonian path in the graph $G$ (see [9]). Such a path contains exactly the required number $\left|Y_{0} \cup Y_{1}\right|-1=2^{k}-1$ of edges $\left\{10 y_{0}, 11 y_{1}\right\}$, each corresponding to a basis vector $\left\{y_{0} \oplus y_{1}, y_{0}, y_{1}\right\}$.

Suppose we have found a set $B$ of $2^{k}-1$ vectors corresponding to the edges in a Hamiltonian path. Since a path is acyclic, any non-empty subset of vectors in $B$ induces a subgraph with at least one vertex of degree 1 . Such a vertex is a position which is in the support of the subset vector sum, and furthermore, will remain so under the addition of any vectors in $B_{k}^{\prime}$, which have no support in $Y_{0} \cup Y_{1}$. This proves that if $B_{k}$ is a basis of $C_{k}$, then $B_{k+1}$ is a basis of $C_{k+1}$, as desired. 
Table 1

\begin{tabular}{llllllllllllllllll}
\hline Position & 0001 & 0010 & 0011 & 0100 & 0101 & 0110 & 0111 & 1000 & 1001 & 1010 & 1011 & 1100 & 1101 & 1110 & 1111 \\
\hline Color & 1 & 2 & 1 & 2 & 3 & 0 & 2 & 0 & 0 & 0 & 0 & 0 & 0 & 0 & 0 \\
Degree & 0 & 1 & 1 & 2 & 1 & 1 & 2 & 2 & 2 & 1 & 2 & 2 & 2 & 2 & 1 \\
Total & 1 & 3 & 2 & 4 & 4 & 1 & 4 & 2 & 2 & 1 & 2 & 2 & 2 & 2 & 1 \\
\hline
\end{tabular}

For $k=1,2,3$, we use the following Hamiltonian paths:

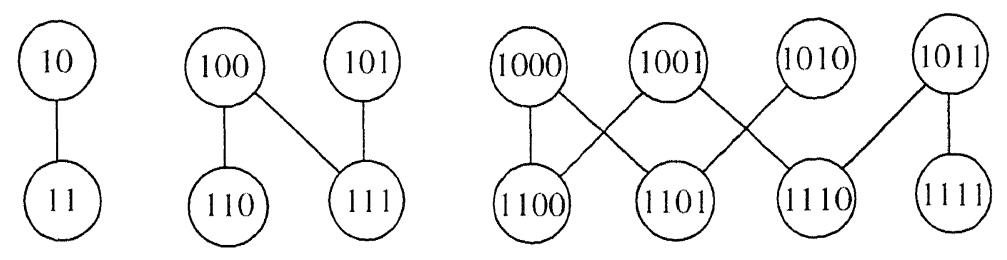

Table 1 gives the number of ones in each position of $B_{4}$, as the total number of basis vectors in which it appears as either a color $y_{0} \oplus y_{1}$ or as a vertex $y_{i}$ (its degree in the Hamiltonian path).

Since the maximum degree in a Hamiltonian path is 2 , the width in positions $1\{0,1\}^{k-1}$ of any $B_{k}$ will be at most 2 . For $k \leqslant 4$, the table shows that the width in positions $0\{0,1\}^{k-1} \backslash\left\{0^{k}\right\}$ of $B_{k}$ is at most 4 . In order to continue our induction beyond $k=4$, it suffices to find a Hamiltonian path in which each color $x \in X$ appears at most twice. Equivalently, we need to find a Hamiltonian cycle in which each $x \in X$ colors exactly two edges. The reason we make the first 3 induction steps explicit is that such a Hamiltonian cycle does not exist in the complete bipartite graph on $\{1000,1001,1010,1011\} \cup\{1100,1101,1110,1111\}$. Instead we compensated for the triple use of the color 0101 in the third path by limiting the degree of node 5 to 1 in the second path.

\subsection{Hamiltonian cycles}

We turn to algebra to find the paths with the required color restrictions.

Let $G F(2)[x]$ denote the class of binary polynomials, that is, with coefficients 0 or 1 , and addition and multiplication $\bmod 2$. We borrow a result from finite field theory which says that for any $k$, there exists a primitive binary polynomial $f(x)$ of degree $k$. This means that $G F(2)[x] /(f)$, the class of residues modulo $f$, is a finite field whose multiplicative group is generated by $x$. In other words, the set $\left\{x^{0}, x^{1}, \ldots, x^{2^{k}-2}\right\}$ contains all $n=2^{k}-1$ non-zero elements.

We can bring $G F(2)[x] /(f)$ in one-one correspondence to each of the three position sets $X, Y_{0}$ and $Y_{1}$ in the inductive step from $k+1$ to $k+2$, where they each have size $2^{k}$. A position $p=p_{1} \ldots p_{k+2}$ will correspond to the binary polynomial $\sum_{i=0}^{k-1} p_{k+2-i} x^{i}$, i.e. we ignore the two first bits that distinguish between $X, Y_{0}$, and $Y_{1}$. For example, with $k=4,101101 \in Y_{0}$ corresponds to $x^{3}+x^{2}+1$. 
Let $(x+1)^{-1}$, the inverse of $x+1$, be equal to $x^{r}$ for some $r, 0 \leqslant r<2^{k}-1$. Note that $x^{i} \mapsto \sum_{j<i} x^{j}=\left(x^{i}+1\right)(x+1)^{-1}=x^{r}\left(x^{i}+1\right)$ is a bijection from all the non-zero elements of $G F(2)[x] /(f)$ to all elements except $x^{r}(0+1)=x^{r}$. Also, $\sum_{j<n} x^{j}=x^{r}\left(x^{n}+1\right)=x^{\prime}(1+1)=0$.

These facts are the basis of the following cycle decomposition (using $\sum^{i}$ as a shorthand for $\sum_{j=0}^{i} x^{j}$ ):

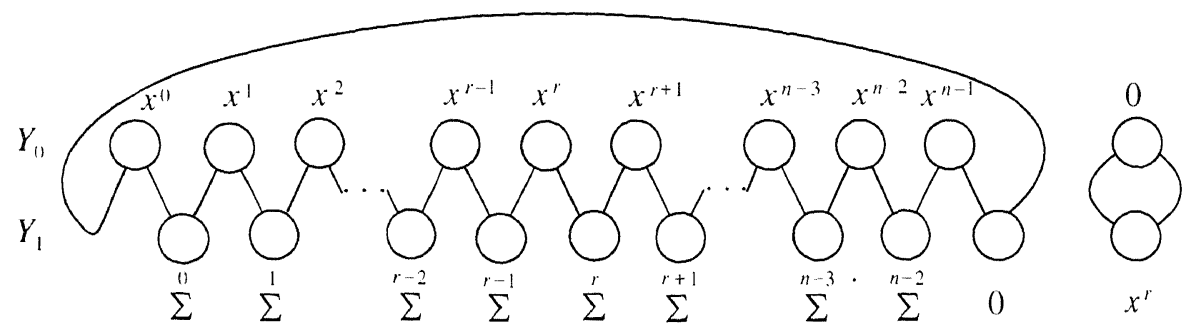

The left cycle uses every color $\sum^{i}, 0 \leqslant i<n$ exactly twice, once on the edge between $\sum^{i-1}$ and $x^{i}$, and once on the edge between $x^{i+1}$ and $\sum^{i+1}$. The right cycle uses the single color not expressible as $\sum^{i}$, namely $x^{r}$, exactly twice. A series of 5 edge swaps transform the two cycles into the following Hamiltonian cycle:

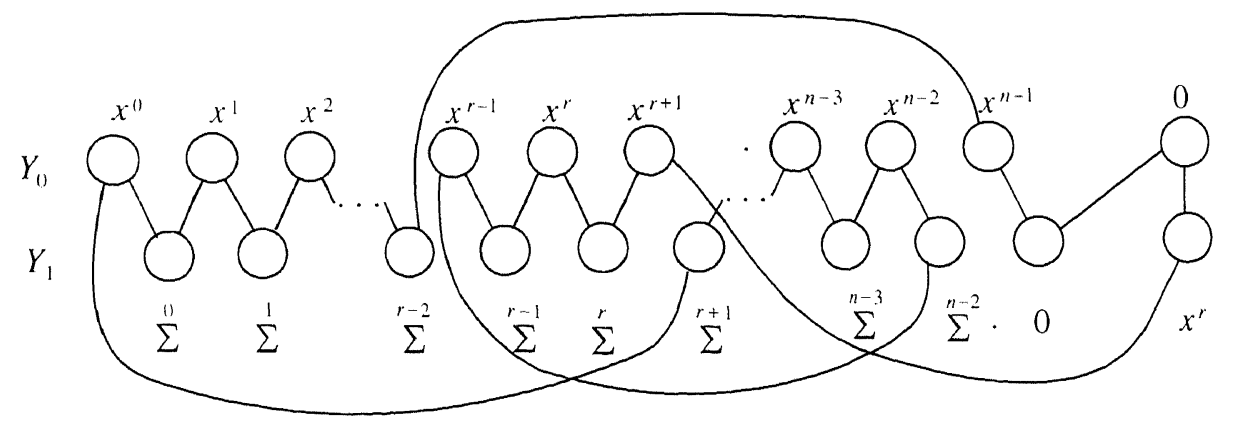

We will refer to the 2-cycle decomposition as 2-cycle and to the Hamiltonian cycle as 1 -cycle. The edge between $x^{0}=1$ and 0 in the 2-cycle has color 1 , as does the edge between $x^{r+1}$ and $x^{r}$ in the 1-cycle, since $x^{r+1}+x^{r}=x^{r}(x+1)=1$. The edge between $\sum^{r-2}$ and $x^{r-1}$ in the 2-cycle has color $\sum^{r-1}$, as does the edge between $x^{0}=1$ and $\sum^{r+1}$ in the 1-cycle, since $\sum^{r+1}+1=\sum^{r-1}+x^{r+1}+x^{r}+1=\sum^{r-1}$. The edge between $x^{r+1}$ and $\sum^{r+1}$ in the 2-cycle has color $\sum^{r}$, as does the edge between $\sum^{r-2}$ and $x^{n-1}$ in the 1-cycle, since $\sum^{r-2}+x^{n-1}=\sum^{r}+x^{r}+x^{r-1}+x^{-1}=$ $\sum^{r}+x^{-1}\left(x^{r+1}+x^{r}+1\right)=\sum^{r}$. The edge between $\sum^{n-2}$ and $x^{n-1}$ in the 2-cycle has color $\sum^{n-1}=0$, as does the edge between 0 and 0 in the 1-cycle. The edge between 0 and $x^{r}$ in the 2-cycle has color $x^{r}$, as does the edge between $x^{r-1}$ and $\sum^{n-2}$ in the 1-cycle, since $x^{r-1}+\sum^{n-2}=x^{r-1}+x^{-1}=x^{r}+x^{-1}\left(x^{r+1}+x^{r}+1\right)=x^{r}$.

It remains to show that this transformation does not suffer from $r$ being too close to 0 or $n-1$. Indeed, $x^{r+1}+x^{r}+1=0$ implies that $r+1 \geqslant k \geqslant 3$, hence $r-1>1$ 
and we are safe on the left. Similarly, $x^{n-r}+x+1=\left(x^{r}\right)^{-1}+x+1=0$ implies that $n-r \geqslant k \geqslant 3$, hence $r+1 \leqslant n-2$, so we are safe on the right too.

Altogether, this shows

Theorem 1. For any $k, C_{k}$ has a $(3,4)$-basis.

An $n$-dimensional hypercube of cliques is the cross product of an $(n-\lfloor\log r\rfloor)$ dimensional hypercube and a complete graph with $2^{\lfloor\log r\rfloor}$ nodes. By Theorem 1, we have

Corollary 2. There is a one-to-one embedding of a hypercube of cliques in a samesized hypercube with dilation 3 and congestion 10.

Proof. See [6].

\section{On $(3,3)$ bases}

In this section we give a sufficient condition for the existence of a $(3,3)$-basis for $C_{k}$. Suppose some degree $k$ primitive polynomial $h(x)$ is the gcd of a trinomial $f(x)=1+x^{j}+x^{m}$ and $x^{n}+1$. Then $C_{k}$ has a $(3,3)$-basis, constructed as follows. Consider the $n \times n$ circulant matrix $F$ generated by $f$; the $i$ th column $F_{i}$ of this matrix $(i=0, \ldots, n-1)$, is formed by the coefficients of $x^{i} f(x) \bmod x^{n}+1$. For example, with $n=7, h(x)=f(x)=1+x+x^{3}$ generates the matrix

$$
F=\left(\begin{array}{lllllll}
1 & 0 & 0 & 0 & 1 & 0 & 1 \\
1 & 1 & 0 & 0 & 0 & 1 & 0 \\
0 & 1 & 1 & 0 & 0 & 0 & 1 \\
1 & 0 & 1 & 1 & 0 & 0 & 0 \\
0 & 1 & 0 & 1 & 1 & 0 & 0 \\
0 & 0 & 1 & 0 & 1 & 1 & 0 \\
0 & 0 & 0 & 1 & 0 & 1 & 1
\end{array}\right) .
$$

We use the fact that $h(x)$ is primitive to define a column reordering of $A_{k}$, called $A_{k}^{\prime}$, whose $i$ th column corresponds to $x^{i} \bmod h(x)$. Now $A_{k}^{\prime} F_{i}$ corresponds to $x^{i} f(x) \bmod$ $x^{n}+1 \bmod h(x)=x^{i} f(x) \bmod h(x)=0$, since $h(x)$ divides both $f(x)$ and $x^{n}+1$. Thus, all columns of $F$ are in the nullspace $C_{k}^{\prime}$ of $A_{k}^{\prime}$.

From a theorem of König and Rados [7], it follows that the rank of $F$ is $n-$ $\operatorname{deg}\left(\operatorname{gcd}\left(f(x), x^{n}+1\right)\right)=n-\operatorname{deg}(h(x))=n-k$. Now if some column $i$ is linearly dependent on columns $0, \ldots, i-1$, then, since $F$ is circulant, column $i+1$ is linearly dependent on columns $1, \ldots, i$ and therefore also on columns $0, \ldots, i-1$. Similarly, columns $i+2, \ldots, n-1$ would be linearly dependent on the first $i$ columns. Thus, the 
Table 2

Trinomials $f(x)=x^{m}+x^{j}+1$ that imply the existence of (3,3)-bases

\begin{tabular}{|c|c|c|c|c|c|c|c|c|}
\hline$k$ & $m$ & $j$ & $k$ & $m$ & $j$ & $k$ & $m$ & $j$ \\
\hline 8 & 13 & 1 & 67 & 76 & 29 & 120 & 174 & 31 \\
\hline 12 & 19 & 6 & 69 & 75 & 23 & 122 & 128 & 15 \\
\hline 13 & 16 & 3 & 70 & 82 & 15 & 125 & 128 & 3 \\
\hline 14 & 17 & 2 & 72 & 93 & 7 & 126 & 141 & 70 \\
\hline 16 & 29 & 6 & 74 & 80 & 39 & 128 & 131 & 50 \\
\hline 19 & 22 & 3 & 75 & 77 & 4 & 131 & 138 & 61 \\
\hline 24 & 55 & 6 & 76 & 88 & 43 & 133 & 136 & 43 \\
\hline 26 & 29 & 12 & 77 & 80 & 9 & 136 & 139 & 30 \\
\hline 27 & 29 & 1 & 78 & 89 & 2 & 138 & 183 & 23 \\
\hline 30 & 41 & 12 & 80 & 83 & 23 & 139 & 142 & 3 \\
\hline 32 & 59 & 29 & 82 & 85 & 19 & 141 & 148 & 71 \\
\hline 34 & 37 & 6 & 83 & 85 & 14 & 143 & 147 & 1 \\
\hline 37 & 43 & 4 & 85 & 93 & 28 & 144 & 159 & 14 \\
\hline 38 & 42 & 1 & 86 & 91 & 22 & 146 & 149 & 6 \\
\hline 40 & 43 & 3 & 88 & 154 & 37 & 147 & 149 & 19 \\
\hline 42 & 51 & 7 & 90 & 111 & 28 & 149 & 151 & 2 \\
\hline 43 & 53 & 2 & 91 & 99 & 13 & 152 & 155 & 38 \\
\hline 44 & 52 & 15 & 92 & 103 & 39 & 154 & 157 & 22 \\
\hline 45 & 59 & 12 & 96 & 123 & 1 & 155 & 158 & 75 \\
\hline 46 & 58 & 9 & 99 & 101 & 13 & 156 & 188 & 59 \\
\hline 48 & 70 & 27 & 101 & 103 & 2 & 157 & 164 & 25 \\
\hline 50 & 54 & 7 & 102 & 115 & 3 & 158 & 167 & 54 \\
\hline 51 & 53 & 4 & 104 & 109 & 9 & 160 & 177 & 19 \\
\hline 53 & 61 & 28 & 107 & 109 & 8 & 162 & 166 & 27 \\
\hline 54 & 93 & 23 & 109 & 118 & 21 & 163 & 171 & 70 \\
\hline 56 & 67 & 31 & 110 & 117 & 19 & 164 & 189 & 68 \\
\hline 59 & 61 & 26 & 112 & 133 & 1 & 165 & 173 & 42 \\
\hline 61 & 66 & 17 & 114 & 118 & 7 & 166 & 186 & 53 \\
\hline 62 & 77 & 30 & 115 & 125 & 6 & 168 & 179 & 38 \\
\hline 64 & 74 & 21 & 116 & 136 & 1 & 171 & 173 & 10 \\
\hline 66 & 83 & 20 & 117 & 123 & 31 & & & \\
\hline
\end{tabular}

first $n-k$ columns of $F$ must actually be linearly independent, else the rank of $F$ would be less than $n-k$. This shows that $F_{0}, \ldots, F_{n-k-1}$ forms a basis of $C_{k}^{\prime}$, and, by an appropriate permutation of dimensions, a basis of $C_{k}$.

The existence of degree $k$ primitive polynomials $h(x)$ that are the gcd of a trinomial $f(x)=1+x^{m}+x^{j}$ and $x^{n}+1$, is demonstrated in Table 2 for $k \leqslant 171$. Only those $k$ for which there is no primitive trinomial of degree $k$ are listed; see Stahnke [11] for a table of primitive binary polynomials up to degree 171. Therefore, we pose the following:

Conjecture 1. There always exists a trinomial $f(x)$ such that $\operatorname{gcd}\left(f(x), x^{2^{k}-1}+1\right)$ is a primitive polynomial of degree $k$ over $G F(2)$, for any $k$. Consequently, any $C_{k}$ has a (3,3)-basis.

The subsequent effort by [4] shows the conjecture to hold through all $k \leqslant 500$. 


\section{Acknowledgements}

The authors would like to thank Dan Pritikin and Gary Mullen for the careful reading of the manuscript and helpful suggestions.

\section{References}

[1] W. Aiello and T. Leighton, Coding theory, hypercube embeddings, and fault tolerance, in: Proc. $3 r d$ Ann. ACM Symp. on Parallel Alyorithms and Architectures (1991) 125-136; IEEE Trans. Computer, to appear.

[2] E. Assmus, Jr and H. Mattson, On tactical configurations and error-correcting-codes, J. Combin. Theory 2 (1967) 243-257.

[3] L. Babai, H. Oral and K. Phelps, Eulerian self-dual codes, SIA M J. Discrete Math. 7 (1994) 325-330.

[4] I.F. Blake, S. Gao and R.J. Lambert, Construction and distribution problem for irreducible trinomials over finite fields, in: Proc. Holloway Conf. on Finite Fields (Oxford Univ. Press, Oxford, 1995), to appear.

[5] R.H. Hamming, Error detecting and error correcting codes, Bell System Tech. Journal 29 (1950) $147-$ 160.

[6] T. Leighton, Introduction to Parallel Alyorithms and Architectures: Arrays - Trees - Hypercubes, Ch. 3 (Morgan Kaufmann, San Mateo, CA, 1992) 430.

[7] R. Lidl and H. Niederreiter, Finite Fields (Addison-Wesley, CA, 1983)

[8] V. Pless, Introduction to the Theory of Error-Correcting-Codes (Wiley, New York, 1989).

[9] D. Pritikin, Graph embeddings from Hamming bases, DIMACS Workshops on Interconnection Networks and Mapping and Scheduling Parallel Computations, February 1994.

[10] A. Reddy, Parallel input/output architectures for multiprocessors, Ph.D. Dissertation. Dept. of Electronical and Computer Engineering, Univ. of Illinois, Urbana, 1990.

[11] W. Stahnke, Primitive binary polynomials, Math. Comput. 124 (1973) 977-980.

[12] L. Zhang, A new bound for the width of Hamming codes, in: Proc. 6th Internat. Conf. on Computiny and Information, 1994, to appear. 